\title{
Use of Synthetic Aperture Radar images for Crisis Response and Management
}

\author{
Gerardo Di Martino, Antonio Iodice, Daniele Riccio, Giuseppe Ruello \\ Department of Biomedical, Electronic and Telecommunication Engineering \\ University of Napoli Federico II \\ Napoli, Italy
}

\begin{abstract}
Remote sensing provides powerful instruments for the extraction of physical information whose knowledge can be used for supporting the crisis management activities. The use of this huge amount of information is still limited due to the lack of reliable interpretation models that allow to produce value added map of easy use in actual cases. The development of physical models along with the identification of the user needs allow to fill this gap. This paper is focused on the use of Synthetic Aperture Radar data, that provide a lot of information useful in many applications related to natural (volcanoes, earthquakes, floods, and so on) and human-driven (oil spills, Earth, air and sea pollution, and so on) disasters. The actual SAR technology allows a low cost use with significant performances in terms of spatial resolution, temporal revisit time and coverage. The proposed approach is supported by examples that show the potentialities of the use of SAR data for crisis prevention, management and response.
\end{abstract}

Keywords: Synthetic Aperture Radar; crisis management.

\section{INTRODUCTION}

The availability of information in crisis situation is crucial for an effective reaction to the triggering event. If the phenomenon affects a wide area, the crisis managers can be supported in the comprehension of the situation and in the decision making process by updated geospatial information.

Despite remote sensing is one of the most powerful technologies for these purposes, its use is still very limited in actual cases. Available satellite data offer detailed information on wide areas with low revisit time. Such an information could be used in the prevention of crisis as well as for reacting to its consequences. Anyway, the gap between the community of remote sensing products developers and the potential users is still too wide. In this work we present an approach devoted to fill this gap.

As a consequence of the huge potentialities outlined above, the international community implemented various actions aimed at supporting research and application of remote sensing techniques for disaster monitoring and mitigation. The United Nations programs UNOSAT [1] and ReliefWeb [2], and the European Union program GMES [3] are major examples of the increasing interest of international institutions in the use of remote sensing for crisis management and humanitarian projects. In this framework many civilian governmental satellite operators and space agencies joined the International
Charter "Space and Major Disasters" [4], in order to coordinate the efforts toward early disaster response.

In the frame of the above mentioned technical and political context, significant efforts are made by the remote sensing community toward the development of models and techniques able to cope with new systems and technologies.

The paper is mainly focused on the case of Synthetic Aperture Radar (SAR) data. SAR is an extremely powerful instrument, because it allows the acquisition of information in any moment of the day and with any weather conditions. In fact, SAR sends an electromagnetic microwave pulse that can penetrate the clouds and is almost insensitive to the rain, and it measures the electromagnetic field scattered by the illuminated surface.

In this paper we present potentialities and limitations of the SAR data, proposing a conceptual framework for their use at almost no-cost for the beneficiaries in case of crisis. In Section 2 we recall the main characteristics of the SAR data, whose interpretation requires the development of physical models. Also the main fields of application and the main actual and future sensors are presented, providing an insight on the variety and the complexity of the actual scenario. Section 3 is devoted to demonstrate that the actual system development and the actual available data allows an immediate use of SAR data for crisis response and management. The section is completed with meaningful examples of the use of high resolution SAR sensors in actual cases. The last section is devoted to the final discussion.

\section{SAR DATA}

A SAR is a side-looking sensor that measures the reflectivity of the observed scene at microwave frequencies. The reflectivity function, which is strongly related with the sensor geometry of acquisition, carries information on the geometric (microscopic and macroscopic roughness) and the dielectric (electric permittivity and conductivity) properties of the observed surface.

As stated before, SAR data provide a huge amount of information in many crisis situations thanks to their all-weather all-time acquisition capabilities. In the last decade SAR systems technologies have undergone a great leap forward in terms of both spatial resolution and temporal resolution (revisit time). In fact, nowadays the new generation of very high 
resolution satellite SAR sensors, such as TerraSAR-X and Cosmo-SkyMed, which can reach a spatial resolution of $1 \mathrm{x} 1$ $\mathrm{m}^{2}$ in Spotlight mode, can observe the Earth at the spatial scales typically affected by natural and human-driven disasters. Moreover, the use of satellites constellations caused a significant decrease in visit and revisit time, which is a key feature for disaster early response.

TABLE I

MAIN SAR SATELLITES

\begin{tabular}{cccc}
\hline \hline Satellite & $\begin{array}{c}\text { Spatial resolution } \\
\text { (azimuth x ground range) } \\
{[\mathrm{m} \times \mathrm{m}]}\end{array}$ & $\begin{array}{c}\text { Average } \\
\text { revisit time } \\
\text { [days] }\end{array}$ & $\begin{array}{c}\text { Operating } \\
\text { band }\end{array}$ \\
\hline Envisat & $4 \times 20$ & 7 & $\mathrm{C}$ \\
Radarsat-2 & $3 \times 3$ & 0.5 & $\mathrm{C}$ \\
TerraSAR-X & $1 \times 1$ & 3 & $\mathrm{X}$ \\
Cosmo- & $1 \times 1$ & 0.2 & $\mathrm{X}$ \\
SkyMed & $6 \times 6$ & 3.2 & $\mathrm{~S}$ \\
NovaSAR-S & $5 \times 5$ & 3 & $\mathrm{C}$ \\
Sentinel 1 & & &
\end{tabular}

In Tab. I we provide a list of the main SAR satellites with their resolution, revisit time and operating band. Note that the two last satellites are not already in orbit, but their launch is planned in the next 2 years. The very low revisit time of Cosmo-SkyMed is due to its constellation operating mode, where 4 satellites presenting the same technical characteristics work as a unique system in order to provide very short revisit intervals. Moreover, note that the revisit times reported in Tab. I are average ones, and are relevant to the operational mode of maximum spatial resolution of the sensor and in particular areas of the globe (typically in mid latitude areas). Indeed, a short revisit time is essential for the monitoring of disasters, such as flooding or eruptions, whose evolution can spam also several days.

As a matter of fact, the availability of very high resolution SAR data opened the way to the development of techniques not easily implementable before. For instance, the monitoring of flooding in urban areas is often impossible with low resolution data, due to the complexity of the geometry of men made areas. Recently, some studies are focusing on this topic, see for example [5] and [6], where the focus is both on high resolution and real-time processing. However, the great majority of disaster monitoring techniques are based on change detection algorithms, which is aimed at identifying the affected area through a more or less complex elaboration of at least a couple of pre- and post-event images. The change detection can be applied to different quantities related to the original SAR image: the amplitude or intensity, filtered or elaborated versions of the amplitude. For instance the authors developed a method based in whose framework the change detection is applied to quantities related to the fractal parameters of the observed surface [7].

Very often an adequate management of crisis situations would require the use of remote sensing data coming from different sources, e. g. optical data can be effectively used in conjunction of SAR data for planning the logistics of relief actions on field or for observing site characteristics complementary to those observed through radar data. This requires the development of data fusion strategies [8] and the use of adequate Geographical Information Systems (GIS).

\section{APPROACH AND RESULTS}

A SAR image is a map of the scene reflectivity, whose characteristics depend in non-linear way by the physical parameters of the scene and whose interpretation is hard for non-expert users.
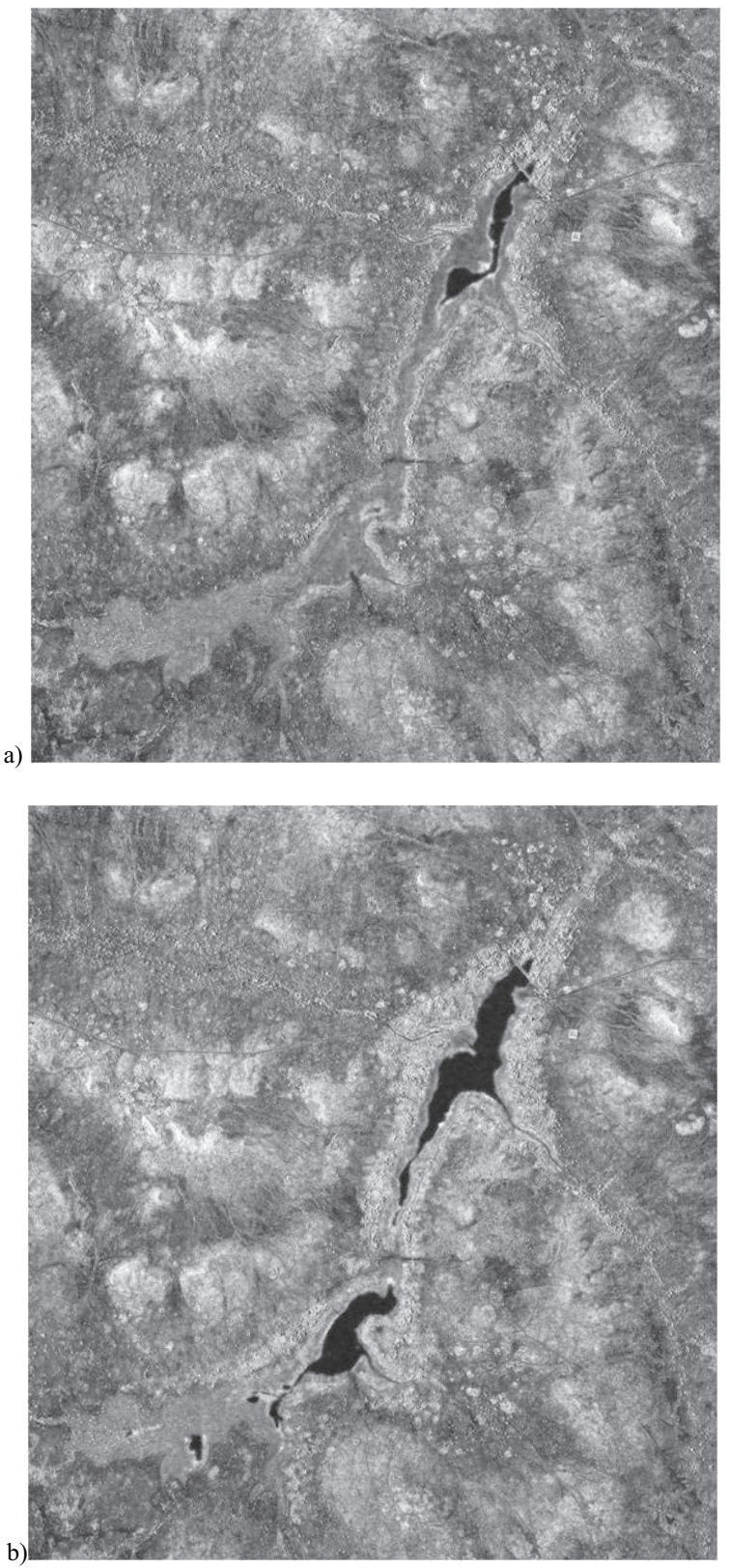


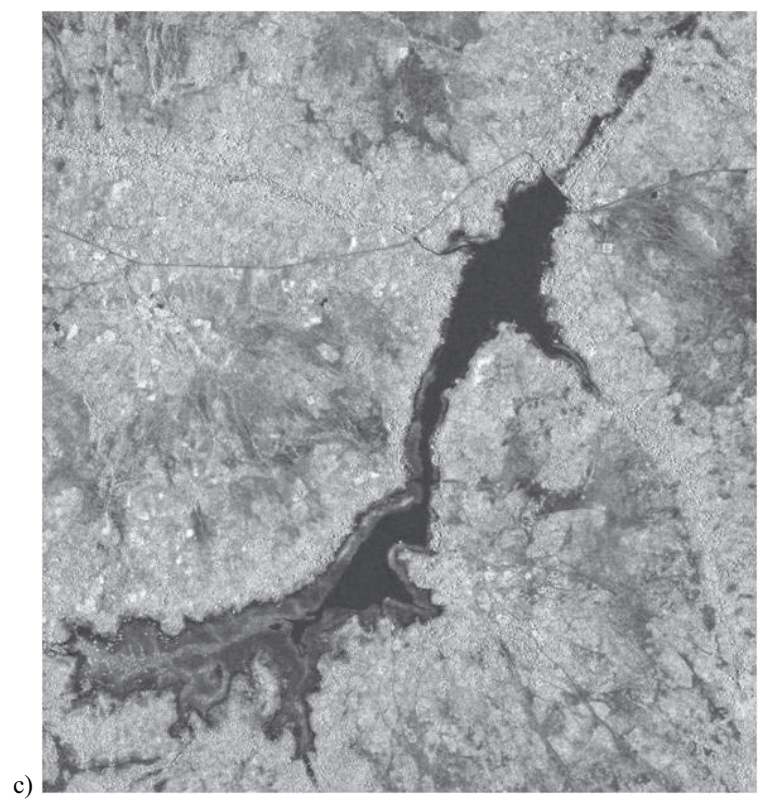

Figure 1. Example of an image of the Tougou dam, Burkina Faso. Pixel spacing is $150 \mathrm{~m}$. The area is about $40 \mathrm{~km} \mathrm{x} 40 \mathrm{~km}$. (a) was acquired during the dry season; (b) was acquired during the wet season; (c) was acquired in flood condition.

In Figure 1 we present some examples of SAR images acquired by the Cosmo-Skymed sensor, relative to a rural area of Burkina Faso, in the West Africa Sahel, acquired in three different conditions. The pixel resolution is $3 \mathrm{~m} \times 3 \mathrm{~m}$. The image is strongly multi-looked, due to presentation requirements. The area is characterized by a big water reservoir, with an availability of surface water that is strongly related to the rain and the evaporation rate.

As it appears clear, Figure 1a refers to a dry season acquisition, when the presence of water is limited to a limited area, identified by black pixels. Figures $1 \mathrm{~b}$ and $1 \mathrm{c}$ refer to a post-rain situation, with the presence of flooded areas.

The possibility of quantitatively exploit the data depends on the capacity of providing from the image a value added map with easy interpretation key. This goal can be reached only under the following conditions:

1. A strong integration between remote sensing experts and application experts;

2. The development of reliable interpretation models, that allow to correctly provide a relation between the acquired image and the observable phenomenon.

In the following, we present an approach devoted to provide this link between SAR experts and potential users. The approach is explained with the development of a case study. The extraction of information from the SAR image must rely on a physical approach, based on three main steps: the image filtering; the image segmentation; a value added map production.

Images are characterized by the speckle, whose interpretation can be crucial for extracting the physical values from the image. The speckle is the typical SAR signal related with the coherent nature of the sensor. Often, filtering has the main goal of removing the speckle from the mean signal. In this paper the filtering is performed with the help of a multitime approach. Anyway, different filters have been developed and can be used. A complete discussion on the choice of the filter goes beyond the goal of the paper.

The image segmentation is often based on empirical or statistical instruments. In this paper we propose a physical based segmentation approach, related on the physical phenomenon that is under observation.

After segmentation, value added map can be produced only after a deep interaction with the applicative people.

The above presented steps are here explained with the aid of a case study. A typical case of disaster monitoring is the identification of the flooded area or the identification of isolated villages or people. The chosen area, presented in Figure 1, is characterized by a rainy and a dry season, with extremely variable land conditions. Therefore, it can be a sort of canonical case for showing the approach, the SAR potentiality and the possible presentation of the results. Of course, any case should be then faced in function of the context where it takes place.

In case of flooding, part of the areas covered by the soil are invaded by water flows, changing both the surface roughness and the dielectric properties of the observed materials. It is already known that the dominant effect is the reduction of reflectivity, due to the fact that most of the incident energy is scattered in specular direction.

The effect on the image is the appearance of dark areas, as it can be seen by the comparison of the images in Figure 1. The knowledge of the physical phenomenon allows the interpretation of the images and the development of a strategy of classification.

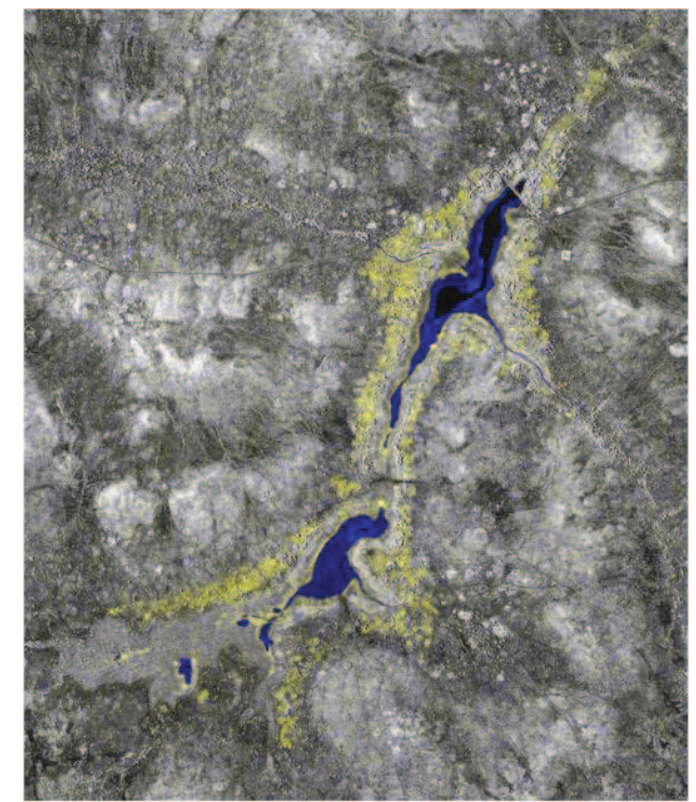

Figure 2. Change detection results relative to the images presented in Figures $1 \mathrm{a}$ and $1 \mathrm{~b}$. 
In the following, we present two possible approaches, based on two different hypothesis. In a first scenario, we assume to face with an early warning problem. In a second case, we assume to deal with a monitoring problem. The two cases are developed with the same set of images, in order to stress the similarities and differences.

\section{A. Early warning}

In a first scenario, we use a couple of images acquired during the rainy and the dry seasons in Burkina, assuming that they can represent the situation before and after a crisis due to a flooding. Due to the high evaporation rate, the surface water occupation is dramatically changed between the two dates. A filtering process and a reliable image registration allow the comparison of the two images and the identification of the flooded areas. In Figure 3, the result of a change detection algorithm based on the image intensity comparison is presented. The black pixels refer to the areas where the water is present in both the acquisitions; blue pixels represent the flooded area. Such an image can be easily read by any user, providing an easy interpretation of the post-event situation.

In order to better appreciate the detail of the available high resolution data, we present a detail of the result in Figure 3. The presented zoom emphasizes the capability of actual SAR sensors of identifying structures of few meters. Also isolated trees can be recognized in the image, without the support of an expert user.

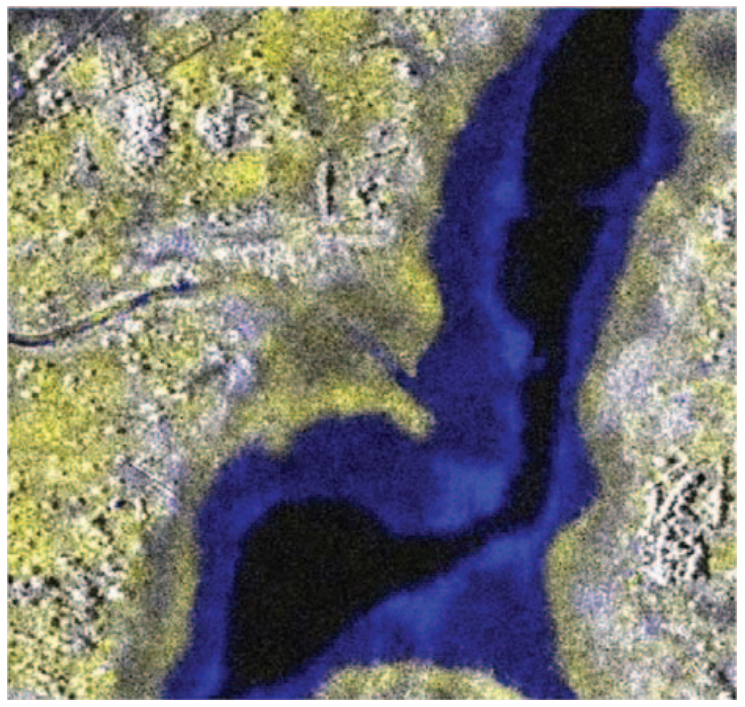

Figure 3. Change detection results relative to the images presented in Figures 1a and $1 \mathrm{~b}$. A detail.

\section{B. Event monitoring}

Some crisis situations can evolve in time, creating the needs of a continuous monitoring of the affected scene. We assume to work in an such a condition, where the situation must be continuously monitored. In this case, thank to the low revisit time of the actual available sensor, we can compare a set of images, relative to the area in evolution.
In this case, we produce false color images, where the evolution of the area occupied by water can be followed during the whole evolution of the event by any user.

In Figure 4 we present an example of the above presented scenario, where the three images of Figure 1 are considered as the evolution in short time of an hypothetic flooding.

The pixels relative to areas always covered by water are presented in black; the pixel flooded before the acquisition relative to Figure $1 \mathrm{~b}$ are represented in blue; violet areas represent the extent of the area affected by the water between the dates of the acquisitions $1 \mathrm{~b}$ and $1 \mathrm{c}$.

The map can be easily readable and clearly identifies the most affected areas. A responsible for the crisis management could use the map for deciding the organization of the first aid, the identification of isolated villages, identification of the roads and so on. Again, in Figure 5, we present a detail of the produced map, in order to present the available spatial detail.

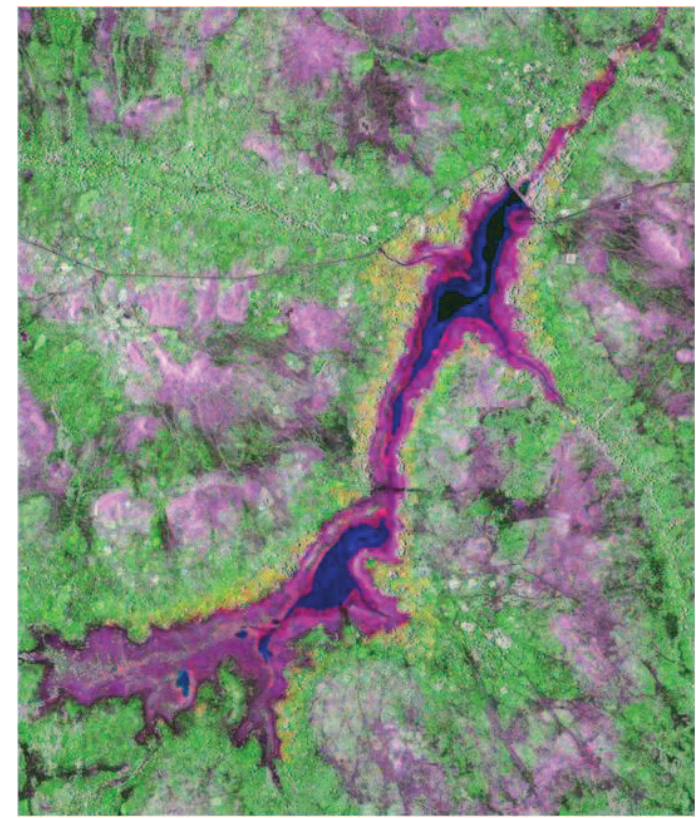

Figure 4. Monitoring of a flooded area, relative to the images presented in Figures $1 \mathrm{a}, 1 \mathrm{~b}$ and $1 \mathrm{c}$.

\section{CONCLUSIONS}

The actual SAR technology is mature for being intensively used in crisis response and management. The available spatial and temporal resolutions guarantee the opportunity of obtaining powerful information to people responsible of the crisis management. The geometrical and dielectric properties of the observed surfaces can provide information on the land cover and the land use; monitoring of volcanoes can be supported by SAR data, as well as flooding, sea and land pollution and many other situations of crisis induced both by natural or human-driven causes. The already developed projects and experiences demonstrated that valuable information can be provided also in low-income countries. In 
all the cases, it is strongly required that the applications are developed with a strong interaction between SAR experts and potential users.

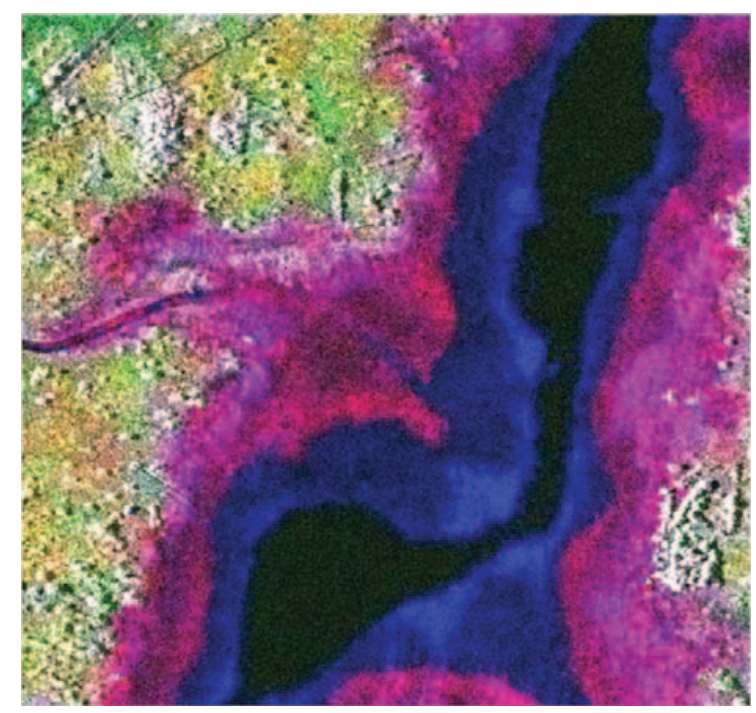

Figure 5. Monitoring of a flooded area, relative to the images presented in Figures $1 \mathrm{a}, 1 \mathrm{~b}$ and $1 \mathrm{c}$. A detail

\section{REFERENCES}

[1] UNOSAT. [Online]. Available: http://unosat.cern.web.ch

[2] ReliefWeb. [Online]. Available: http://reliefweb.int

[3] GMES. [Online]. Available: http://www.respond-int.org

[4] International Charter, Space and Major Disasters. [Online]. Available: http://www.disastercharter.org/main_e.html

[5] Mason, D. C., Speck, R., Devereux, B., Schumann, G. J., Neal, J. C., \& Bates, P. D. (2010). Flood Detection in Urban Areas Using TerraSAR-X. IEEE Trans. Geosci. Remote Sens., 48(2), 882-894.

[6] Mason, D. C.; Davenport, I. J.; Neal, J. C.; Schumann, G. J.-P.; Bates, P. D.; , "Near Real-Time Flood Detection in Urban and Rural Areas Using HighResolution Synthetic Aperture Radar Images," IEEE Trans. Geosci. Remote Sens., pp.1-12, doi: 10.1109/TGRS.2011.2178030.

[7] Di Martino, G., Iodice, A., Riccio, D., \& Ruello, G., "A Novel Approach for Disaster Monitoring: Fractal Models and Tools", IEEE Trans. Geosci. Remote Sens., pp. 1559-1570, 2007.

[8] Voigt, S.; Kemper, T.; Riedlinger, T.; Kiefl, R.; Scholte, K.; Mehl, H.; , "Satellite Image Analysis for Disaster and Crisis-Management Support," Geoscience and Remote Sensing, IEEE Transactions on , vol.45, no.6, pp.1520-1528, June 2007. 